\title{
Heart Failure by Non-invasive Diagnostic Technique
}

National Cancer Institute

\section{Source}

National Cancer Institute. Heart Failure by Non-invasive Diagnostic Technique. NCI

Thesaurus. Code C119215.

A finding of heart failure through a non-invasive diagnostic technique. 